\title{
18. Metafore monetarie nella narrativa italiana del tardo Ottocento
}

Igor Tchehoff

Stockholms universitet

\section{Introduzione}

La presente indagine sulle metafore del denaro fa parte di un progetto più ampio dedicato allo studio dei motivi economici ${ }^{\mathrm{I}}$ nel romanzo italiano del secondo Ottocento. Poiché il senso globale di un'opera nasce dall'interazione dei suoi livelli costitutivi, un'analisi più sistematica delle metafore può dare un contributo importante all'interpretazione del testo. Questo vale in particolare per le metafore monetarie, perché il denaro, essendo un mezzo universale di scambio privo di una sua essenza particolare, può prestarsi a una grande varietà di significati metaforici. Soprattutto dagli scrittori veristi il denaro viene raffigurato come una presenza ossessiva in un mondo dove tutti i personaggi adorano "il dio Quattrino" (Chelli), che risulta essere anche "il miglior frutto della loro vita" (Serao). In tale modo le metafore monetarie possono svolgere alcune funzioni di tipo ideologico, conoscitivo e narrativo. Il corpus delle metafore monetarie esaminate in questo studio consiste di tre romanzi pubblicati in Italia nel corso di pochi anni: L'eredità Ferramonti (I884) di Gaetano Carlo Chelli, Mastro-don Gesualdo (I889) di Giovanni Verga e Il paese di cuccagna (I 890) di Matilde Serao.

Gli esempi dell'uso metaforico del denaro saranno analizzati in una prospettiva che combina le teorie linguistiche e cognitive della metafora con un approccio più strettamente letterario. ${ }^{2} \mathrm{Al}$ centro della presente analisi vi saranno le metafore monetarie che mettono in rilievo la circolazione e la tras-formazione del denaro che metaforicamente può passare da uno stato fisico all'altro. Queste caratteristiche del fenomeno in questione sono state indagate attentamente sia dagli scrittori del

Come citare questo capitolo:

Tchehoff, Igor, Metafore monetarie nella narrativa italiana del tardo Ottocento. In: Engwall, Gunnel \& Fant, Lars (eds.) Festival Romanistica. Contribuciones lingüísticas Contributions linguistiques - Contributi linguistici - Contribuições linguísticas. Stockholm Studies in Romance Languages. Stockholm: Stockholm University Press. 20I 5, pp. 346-359. DOI: http://dx.doi.org/Io.I6993/bac.r. License: CC-BY 
secondo Ottocento sia dagli studiosi moderni delle metafore. Si tratta dunque di esaminare in questa sede soprattutto le metafore che mettono in rilievo la circolazione del denaro e non la totalità delle metafore monetarie presenti nel corpus.

Per i romanzieri italiani si tratta di capire e di interpretare la metamorfosi della società tradizionale che veniva trasformandosi con l'avvento della modernizzazione veicolata dai processi monetari. Gli scienziati cognitivisti hanno invece esaminato come le metafore materiali possono rendere più concreti e comprensibili i fenomeni astratti, quale il denaro. Il corpus letterario ottocentesco darà sostegno a entrambe le ipotesi che finora sono state formulate sulle metafore concettuali che governano la metaforizzazione del denaro: la prima, che riguarda l'intera economia sociale ed evidenzia i flussi finanziari (O'Connor I998) e la seconda, che vede il denaro in stretta relazione con le necessità personali di un individuo in carne ed ossa (Casadei I996).

\section{Il quadro teorico}

Per quanto riguarda la prospettiva metodologica, si farà uso delle teorie legate al nome di George Lakoff, che vedono la metafora non come un fenomeno periferico nell'uso del linguaggio, ma come un fattore centrale nella struttura della lingua e della mente, qualcosa che determina la nostra percezione del mondo, aiuta a organizzare le nostre esperienze e di conseguenza determina le nostre azioni. Secondo questo approccio cognitivista, "metaphor resides in thought, not just in words" (Lakoff \& Turner I989: 2).

I sostenitori della Conceptual Metaphor Theory fanno una distinzione tra le metafore concettuali e le metafore linguistiche. ${ }^{3}$ La metafora concettuale è una nozione sistematica che fa parte di una certa cultura e che viene realizzata attraverso varie metafore linguistiche. Per esempio, a una metafora concettuale come "IL TEMPO È UNA RISORSA (economica)" corrispondono in italiano le metafore linguistiche "sprecare il tempo", "fare fruttare il tempo" e "guadagnare tempo". "TIME IS MONEY” è, secondo Lakoff e Johnson, un modo metaforico di concettualizzare il tempo usando le nostre esperienze quotidiane del denaro (I980: 7-9). Una metafora concettuale "CONOSCERE È VEDERE”, invece, stabilisce una relazione tra due campi diversi di esperienze, quali conoscenza e visione (Casadei I996: 76):

Una metafora concettuale è infatti una proiezione (mapping) tra due domini, detti dominio origine (source domain, ad esempio il vedere) e dominio oggetto 
(target domain, ad esempio il conoscere); la proiezione dà luogo a un insieme di corrispondenze sistematiche e coerenti tra elementi del dominio origine ed elementi del dominio oggetto [...] (ibid.).

Generalmente, il dominio origine indicherà un campo più concreto che sarà impiegato per caratterizzare il campo più astratto del dominio oggetto. In questo modo le metafore aiutano a organizzare e concretizzare le nostre conoscenze. Di conseguenza, non dovrebbe sorprendere la massiccia presenza di metafore nei discorsi che riguardano i processi economici, spesso percepiti come complessi e intangibili:

Thus, in THE ECONOMY IS A LIVING ORGANISM metaphor, the economy (target), being a highly abstract and intangible concept, is metaphorically structured in terms of a living organism (source), a concept which we, as human beings, understand and conceive of much more easily (Silaški \& Kilyeni 20II: 63).

Allo stesso tempo si può notare come in ogni metafora concettuale venga compiuta una scelta particolare, avvicinando due campi, o domini, diversi. Alcune caratteristiche del dominio origine saranno attribuite al dominio oggetto, mentre altri tratti del dominio oggetto rimarranno in ombra. Se l'economia viene considerata un organismo vivente e non un insieme di azioni umane, si potrebbe perdere di vista la possibilità di governare politicamente il suo sviluppo e funzionamento. Il compito dei politici diventa piuttosto quello di creare le migliori condizioni per il funzionamento di questo sistema autonomo, senza la possibilità di intervenire direttamente. In questo senso le metafore possono diventare portatrici di una particolare visione del mondo o di un'ideologia.

L'avvicinamento di due campi esperienziali diversi effettuato dalla metafora si trova anche al centro della discussione sulle metafore letterarie e su quelle convenzionali. Gli scrittori e i poeti spesso adoperano metafore per allargare l'universo concettuale del lettore e offrire uno sguardo innovativo sull'esistenza umana. Le metafore che si riscontrano nei testi poetici sembrano più creative rispetto a quelle che noi usiamo quotidianamente, ma sul carattere preciso di questa differenza tra $\mathrm{i}$ due tipi di metafore esistono alcune ipotesi divergenti. Secondo Elena Semino e Gerard Steen (2008), si può distinguere tra gli approcci teorici che stabiliscono una netta distinzione tra le metafore letterarie e non-letterarie, e altre proposte teoriche che enfatizzano elementi di continuità tra l'uso delle metafore nel linguaggio letterario e quello non-letterario. Per gli studiosi appartenenti alla scuola formalista e alla stilistica anglosassone, le metafore poetiche rappresentano un particolare tipo di 
deviazione dalle norme semantiche (2008: 233-234). Per i teorici cognitivisti, invece, l'uso poetico della metafora è visto come una realizzazione innovativa del potenziale concettuale comune alle metafore convenzionali: "though a particular poetic passage may give a unique linguistic expression of a basic metaphor, the conceptual metaphor underlying it may nonetheless be extremely common" (Lakoff \& Turner I989: 50).

\section{Le metafore concettuali del denaro}

Il denaro svolge un ruolo importante nella nostra vita quotidiana, ma allo stesso tempo rimane un fenomeno astratto e complesso. Dal punto di vista teorico, la definizione del denaro si divide in tre parti: è un mezzo di scambio, un'unità di conto e una riserva di valore. Essendo un mezzo di scambio universale, il denaro non possiede delle caratteristiche intrinseche, perché può essere scambiato con una merce qualsiasi, è uno strumento impersonale che si può prestare a qualsiasi scopo. Come riserva di valore, il denaro avrà, comunque, sempre un valore positivo, a dispetto dei teologi medievali e di Lutero che lo vedevano invece come lo sterco del demonio. Questa indeterminatezza del denaro fa parte della sua natura e diventa anche un fattore produttivo, qualcosa che stimola l'immaginario collettivo e individuale e che si rispecchia in numerosi proverbi, aforismi e sentenze che riguardano il denaro.

La necessità di concretizzare questo fenomeno proteiforme ha dato origine alla metafora concettuale "DENARO È MATERIA" che è comune a tante lingue. ${ }^{4}$ Per le metafore monetarie in spagnolo, Kathleen O'Connor ha proposto la seguente classificazione tripartita degli stati fisici del denaro: "Solids are associated with long-term security and indivisibility; liquid, with transferability and instancy of access; and gas, with uncontainability, unpredictability, and loss" (I998: I4I). A queste metafore concettuali si possono facilmente trovare corrispondenti in italiano: "la casa, un solido investimento", "flussi finanziari", "La Borsa di Milano si sgonfia”. Anche se O’Connor sembra basarsi unicamente sui testi specialistici, le stesse metafore concettuali possono essere identificate in un brano de Il paese di cuccagna di Matilde Serao:

Aveva stentato dapprima a comprendere quale sottile elemento dissolvente disperdesse i quattrini della sua casa, [...]: non arrivava a capire per quale ferita piccina, a una a una, stillassero via le goccie del sangue di casa Fragalà. Invano prosperava la bottega, invano ella faceva prodigi di economie, il denaro spariva, spariva, sentendo ella il vuoto sotto la solidità apparente della loro casa commerciale sentendo il languore irrimediabile di un corpo a cui il sangue va mancando (I 890 : I36). 
La scrittrice napoletana costruisce un complicato sistema metaforico. La casa commerciale, prima stabile, perde gradualmente la sua solidità a causa dell'elemento dissolvente del gioco. La scrittrice insiste sulla visione metaforica del denaro come sangue che sparisce goccia a goccia e fa diventare la casa Fragalà simile a un corpo dissanguato. La metafora "IL DENARO È SANGUE", tipica di questo periodo, sarà esaminata in seguito. Inoltre, si può notare che la divisione tripartita proposta da O'Connor per le metafore spagnole di oggi sembra trovare piena conferma in un romanzo italiano del secondo Ottocento. Questo fatto dà sostegno all'ipotesi cognitivista che afferma la continuità tra le metafore convenzionali e quelle letterarie. Infine, accanto alla metafora del sangue/ liquido troviamo "la casa", "la bottega", la "casa commerciale" e "un corpo" - quattro entità che possono essere accomunate sotto la categoria concettuale e ontologica del contenitore. Lo schema del contenitore insieme alla visione del denaro in movimento sorreggono la metafora concettuale "IL DENARO È UN LIQUIDO" (Silaški \& Kilyeni 20I I: 66), che è poi la variante più produttiva tra le metafore monetarie.

Una motivazione differente delle metafore concettuali del denaro è stata suggerita da Federica Casadei. L'approccio della studiosa italiana è molto diverso, perché la sua indagine è incentrata sulla semantica delle espressioni idiomatiche italiane, che vengono ricondotte alle metafore concettuali come intese da Lakoff. Il suo studio non riguarda in particolare le metafore monetarie, ma analizza le espressioni idiomatiche, un alto numero delle quali rimanda alle metafore concettuali del denaro. Un aspetto interessante dell'uso metaforico del denaro che risulta dall'analisi di Casadei, è che "il denaro è una delle poche entità che compaiono nelle e.i. [espressioni idiomatiche] sia come dominio origine che come dominio oggetto di metafore" (I996: 353). L'ubiquità del denaro come categoria mentale può essere notata, per esempio, nelle metafore concettuali "IL TEMPO È UNA RISORSA (economica)" e "IL DENARO È ARIA/OSSIGENO", che appaiono nelle espressioni idiomatiche italiane.

Dal corpus delle espressioni idiomatiche esaminato da Casadei si possono estrarre due gruppi che corrispondono alle seguenti metafore concettuali:

I. IL DENARO È ARIA/OSSIGENO: "avere una/la corda al collo" essere in una situazione economica disperata, "strozzare" - prestare denaro a forte usura, "strozzino/cravattaio" - usuraio, "avere bisogno di ossigeno" - avere bisogno di denaro (I996: $32 I-322)$. 
2. IL DENARO È UN LIQUIDO: “essere una sanguisuga” - essere avido spec. di denaro, fare lo strozzino, "succhiare anche il midollo (a qcn)" - sfruttare fino in fondo spec. economicamente, "avere la borsa asciutta/le tasche asciutte" - non avere denaro; "essere/stare/restare a secco/in secco" - essere senza denaro, “nuotare nell'oro" - essere molto ricco (I996: 309, 353-354).

La metafora concettuale del denaro solido, invece, non trova corrispondenti nel corpus delle espressioni idiomatiche italiane di Casadei. Ciononostante, si possono identificare altre espressioni che si basano sull'immagine del denaro che viene mangiato e che di conseguenza sarebbe solido: "mangia mangia", "mangeria", "mangiasoldi". Un rapido sguardo agli esempi enunciati permette subito di constatare che le motivazioni di queste metafore concettuali del denaro sono molto diverse da quelle proposte da O'Connor per i testi specialistici. Nel corpus delle espressioni idiomatiche le metafore del denaro sono in stretto rapporto con il corpo dell'individuo e i suoi bisogni vitali. Secondo Casadei, la metafora concettuale "IL DENARO È ARIA/OSSIGENO" è un caso particolare della metafora "LE DIFFICOLTÀ ECONOMICHE SONO MANCANZA D'ARIA/ SOFFOCAMENTO”, che si basa sulla relazione metonimica tra essere in vita e respirare (I996: 32I). "IL DENARO È UN LIQUIDO", invece, è probabilmente motivato da "IL DESIDERIO È FAME/SETE" oltre che dallo schema del contenitore (I996: 353-354).

Si vedono, dunque, due motivazioni molto diverse delle stesse metafore concettuali del denaro. La proposta di O'Connor parte da una visione globale del denaro e del suo funzionamento nell'economia sociale. Nella prospettiva di Casadei, invece, il denaro viene metaforizzato dal punto di vista di un individuo che deve mangiare, bere e respirare. In seguito si vedrà come queste due visioni metaforiche del denaro ricorrono nel corpus delle metafore letterarie del tardo Ottocento.

\section{Il denaro come sangue}

La metafora concettuale "IL DENARO È SANGUE" è un caso particolare di “IL DENARO È UN LIQUIDO". Questa metafora, fondamentale nel brano citato di Matilde Serao, ha goduto di una grande fortuna nel corso della storia grazie alla sua concretezza e capacità esplicativa, probabilmente perché rimanda alla comune esperienza umana del corpo. La dimensione corporea è centrale, secondo la teoria cognitivista, nella 
strutturazione metaforica che sorge dall'esperienza umana (Lakoff $\&$ Johnson I980: II7-II8). Inoltre, si può notare che nella metafora "IL DENARO È SANGUE” il dominio origine, cioè, sangue, può essere considerato, dal punto di vista semantico, una combinazione di due semi presenti nel concetto del denaro: l'idea della circolazione (denaro come mezzo di scambio) + l'idea del valore (denaro come riserva di valore). Il sangue è appunto un liquido in continua circolazione e di altissimo valore per un individuo.

Partendo dallo schema corporeo, la metafora del sangue è stata spesso applicata al denaro dagli studiosi dell'economia politica dell'epoca premoderna. Secondo Michel Foucault, il mercantilismo aveva adottato la metafora della moneta come il sangue della società, incorporando nella sua analisi la scoperta della circolazione sanguigna fatta da William Harvey (Foucault 2006 [I966]: I97). La fortuna di questa metafora tra gli economisti sembra persistere ancora nel Settecento, ma poi cade in disuso nell'ambito specialistico, come sostiene Jerah Johnson, perché l'economia politica si sviluppa come disciplina e diventa più sofisticata col tempo (I966: I22). Per i non-specialisti, invece, la metafora mantiene la sua forza esplicativa. In L'eredità Ferramonti, Chelli spiega il ruolo del credito nel sistema economico a proposito di un istituto finanziario che doveva "cercare la propria clientela nel piccolo commercio, languente nell'anemia del credito, ch'è il suo sangue vitale” (I 884: 73). Il denaro, o il credito bancario è, dunque, il sangue dell'economia e del commercio e la metafora usata da Chelli rispecchia una visione precisa della società e ha un valore conoscitivo. Inoltre, si può notare che, come nella citazione di Serao, la mancanza di denaro viene paragonata al sentimento fisico del languore.

Accanto alla variante economico-politica della metafora concettuale "IL DENARO È SANGUE", il corpus romanzesco contiene esempi di una variante più individuale che concerne la descrizione di alcuni personaggi, soprattutto quando i narratori danno la parola ai protagonisti che si definiscono in tali termini. Mastro-don Gesualdo di Verga dice "[c]ol mio denaro, capite, vossignoria? col sangue mio!" (I 889: 87) e più tardi avrà l'impressione che "gli toglievano il denaro, il sangue delle vene, per tenerlo sottomano, prigioniero" (I 889: 246) Nel romanzo di Serao, un'usuraia, Donna Concetta, “[v] oleva il suo denaro, il sangue suo" (I 890 : I 27). In tal modo il narratore può anche prendere le distanze dalla visione dell'individuo che si identifica con il proprio denaro, che poi è una caratterizzazione sociale dei borghesi o dei popolani arricchiti. La metafora "IL 
DENARO È SANGUE" può dunque essere portatrice non solo di una visione scientifica e concettuale, come si è visto prima, ma anche di una prospettiva sociale ed ideologica. Inoltre, "sangue" come dominio origine in italiano può avere un significato molto ampio, ed essere inteso come "vita", "salute" o fare riferimento ai rapporti famigliari e di parentela. La frase "[c]ol mio denaro [...] col sangue mio" di Mastro-don Gesualdo può essere interpretata nel senso che la caccia al denaro occupa il posto della sfera famigliare nella vita del protagonista, che è un tema ricorrente nei tre romanzi esaminati. Lo scrittore siciliano sfrutta abilmente il potenziale semantico di una metafora convenzionale nella costruzione del romanzo quando Mastro-don Gesualdo alla fine realizza le conseguenze tragiche della visione esistenziale "IL DENARO È SANGUE”. Verga, Serao e Chelli usano metafore di questo tipo nella caratterizzazione dei personaggi, per poi, a livello dell'intreccio, far vedere gli effetti di una visione del mondo che risulta dal significato della metafora. In tal modo, il senso globale dell'opera viene costruito dall'interazione tra il livello metaforico e il livello degli eventi narrativi.

\section{Le due motivazioni della metafora concettuale "IL DENARO É UN LIQUIDO"}

Nella terminologia economica, l'immagine del denaro come liquido è la metafora più frequente. Anche se nella nostra esperienza quotidiana le monete e le banconote hanno una forma tangibile, il denaro in senso astratto e generale viene concepito come un liquido (Silaški \& Kilyeni 20I I: 65). Denaro e liquidi condividono alcune caratteristiche strutturali come omogeneità e facile divisibilità. Nel paradigma fisico la circolazione dell'acqua è determinata dalle leggi naturali e il denaro che circola nella società sembra svolgere la stessa funzione. Il nostro modo moderno di metaforizzare il denaro è sicuramente influenzato dalle nostre esperienze del denaro in forma non-tangibile dei conti bancari, delle carte di credito e delle transazioni elettroniche, ma anche il corpus delle metafore ottocentesche fornisce la stessa immagine. Di conseguenza, la motivazione della metafora concettuale "IL DENARO È UN LIQUIDO" offerta da O'Connor che sottolinea il ruolo della circolazione e della trasferibilità, sembra più in linea con la nostra comprensione moderna del denaro. La motivazione più corporea della stessa metafora nell'ambito delle espressioni idiomatiche, proposta da Casadei che lega "IL DENARO È UN LIQUIDO" alla metafora concettuale 
"IL DESIDERIO È FAME/SETE", può sembrare sorprendente in questo contesto. Probabilmente le espressioni idiomatiche esprimono un sapere più antico e concreto, legato all'epoca premoderna, quando il denaro era un risultato del lavoro fisico e serviva soprattutto a soddisfare le necessità vitali dell'individuo come l'alimentazione. A questo punto si tratta di capire se le due motivazioni della stessa metafora concettuale si autoescludano o possano essere combinate per arricchire la nostra comprensione del fenomeno in questione. I romanzi che ritraggono e problematizzano il passaggio dalla società tradizionale a quella moderna potranno forse fornire una possibile risposta?

Nel corpus romanzesco le realizzazioni concrete della metafora concettuale "IL DENARO È UN LIQUIDO" mostrano una materia sempre in movimento. Verga parla "del denaro a fiumi da intascare" (I 889: 242); “[i]l denaro a fiumi, un va e vieni” ( I 889: 235) e anche nel romanzo di Serao queste metafore sono numerose:

(I) "un affluire magico di denaro che corre al denaro, per incanto" (I890: $72)$,

(2) "L'assistito, $[\ldots]$ pompava denari da tutto il gruppo dei cabalisti" (I890: I04),

(3) "è una gran liquefazione di denaro, come in un crogiuolo, donde fuggisse tutto il metallo" (I 890: I4I),

(4) “l'indomani, sarebbero sgorgati denari, denari, denari" (I890: 7I).

L'ultimo esempio funziona anche al livello iconico dove l'abbondanza si esprime attraverso la ripetizione della parola-chiave "denari”. Queste manifestazioni concrete della metafora concettuale "IL DENARO È UN LIQUIDO" nei romanzi sembrano determinate piuttosto dalla circolazione del denaro e dalla sua trasferibilità, e non dal suo legame al corpo. Dall'altro lato ci sono metafore monetarie che dimostrano come tutta questa circolazione sia messa in moto proprio dai desideri e dalle passioni dei protagonisti:

(5) "succhiare, come un vampiro, sangue e quattrini di quella famiglia" (Chelli I 884: 279),

(6) "la bocca vorace che inghiottiva tutti i denari del vecchio" (Serao I 890: I I 4),

(7) "l'assistito ronzava intorno [...] sempre succhiatore di denari" (Serao I 890: I37),

(8) "Denaro, denaro, era quello che voleva, era la sua sete, era la sua fame, era la sua anima che solo quello chiedeva, quello che solamente chiedeva il suo corpo. Denaro! O sarebbe morto, ecco!” (Serao I890: 198). 
L'ultima citazione di Serao conferma pienamente la motivazione della metafora concettuale proposta da Casadei, in cui la brama di denaro viene metaforizzata in termini di fame e di sete. Le metafore monetarie possono, dunque, essere spiegate in entrambi i modi, sia partendo dalla visione globale del denaro in circolazione, sia considerando il denaro dal punto di vista di un individuo totalmente dominato dalla brama di soldi. Infatti, il romanzo ottocentesco dimostra chiaramente che la circolazione del denaro non è solo un fenomeno oggettivo, mosso dalle leggi naturali com'è il caso della circolazione dell'acqua, ma che i flussi monetari vengono determinati dalle passioni e dai desideri umani che sorgono nel corpo, come risulta dall'interpretazione delle espressioni idiomatiche offerta da Casadei. In questo modo il romanzo compie una sintesi dei due approcci che si sono visti finora. Allo stesso tempo le due motivazioni della metafora concettuale "IL DENARO È UN LIQUIDO" pongono l'accento su due delle definizioni del denaro di cui si è parlato prima, cioè la sua funzione come un mezzo di scambio e quella come una riserva di valore. Anche se le due funzioni sono legate tra loro, la circolazione del denaro viene soprattutto determinata dalla sua scambiabilità, mentre l'oggetto del desiderio è per definizione un oggetto di valore. Infatti, nel romanzo di Verga la brama di accumulazione di cui Mastro-don Gesualdo è vittima riguarda soprattutto i beni materiali o oggetti di valore, come proprietà agricole o la roba in generale, e non tanto il denaro in sé.

Si può constatare che le due motivazioni della metafora concettuale "IL DENARO È UN LIQUIDO" non si contraddicono e combinate insieme possono contribuire a una comprensione maggiore del fenomeno in questione. Cioè che interessa gli scrittori è, appunto, proporre un quadro generale del loro tempo che consiste delle varie prospettive dei singoli protagonisti. Al centro della loro indagine si trova l'interazione dell'individuo con la collettività, un'interdipendenza che, con l'arrivo della società moderna, viene mediata attraverso i meccanismi monetari. La funzione fondamentale del denaro in questo processo di modernizzazione è stata caratterizzata dal filosofo tedesco Georg Simmel che, pochi anni dopo la pubblicazione dei romanzi analizzati, descriveva il denaro nel modo seguente: "In breve, il denaro è l'espressione e lo strumento di un rapporto, della reciproca dipendenza degli uomini, della loro relatività, la quale fa sì che la soddisfazione dei desideri dell'uno dipenda da un altro e viceversa" (cit. in Poggi I998: I42). La soddisfazione dei desideri di cui parla Simmel può, nel caso di alcuni protagonisti come Irene Ferramonti, essere metaforizzata in termini 
conflittuali dalla comunità che sospetta la giovane donna di "succhiare, come un vampiro, sangue e quattrini di quella famiglia" (Chelli I 884: 279). La protagonista stessa descrive la sua interazione con gli altri in modo differente: "L'esistenza è una lotta dell'individuo con la società, piena di varie vicissitudini. Chi perde, è un imbecille se non si prepara a vincere in un assalto successivo" (Chelli I 884: 273).

\section{Prospettive per la ricerca futura}

Per finire, si vorrebbe indicare due indirizzi promettenti per la futura ricerca sulle metafore monetarie nel romanzo italiano dell'Ottocento. Il primo riguarda la dimensione esistenziale e ideologica delle metafore letterarie che può essere esaminata nella prospettiva della teoria di Great Chain of Being, proposta da George Lakoff e Mark Turner. Questa teoria riguarda la nostra comprensione del mondo, "la natura delle cose", ovvero il senso comune che ha una struttura metaforica e che si rispecchia nei modi di dire e nei proverbi: "We have a commonplace theory of forms of being - that they have essences and that these essences lead to the way they behave or function" (I989: I69). Secondo gli studiosi americani, la struttura metaforica essenziale che determina la nostra comprensione del mondo è gerarchica e statica, e porta a delle conseguenze sul piano politico:

The existence of these global and microcosmic hierarchies in the cultural model of the Great Chain, and in its conscious elaborations in the West, has had profound social and political consequences, because the cultural model indicates that the Great Chain is a description not merely of what hierarchies happen to exist in the world but, further, of what the hierarchies in the world should be. This implies that it is wrong to attempt to subvert this order of dominance (1989: 210).

La visione immutabile e pessimista delle cose, che spesso caratterizza il verismo italiano, e che soprattutto nel caso di Verga si riflette nei proverbi e nel senso comune del popolo, potrebbe proficuamente essere analizzata nel quadro della teoria metaforica di Great Chain of Being di Lakoff e Turner.

Un altro interessante indirizzo di ricerca concerne il rapporto tra le metafore poetiche e quelle convenzionali. Le metafore monetarie analizzate finora riguardano soprattutto i vari stati materiali del denaro, solido e liquido, e come tali condividono una comune base concettuale con le metafore convenzionali, pur possedendo, in alcuni casi, una maggiore 
complessità (si veda il brano di Serao in "Le metafore concettuali del denaro"). A questo punto sorgono due domande sul carattere delle metafore monetarie. La prima è se le metafore del denaro possano davvero essere poetiche e innovative, mentre la seconda riguarda il rapporto tra le metafore poetiche e il genere del romanzo verista e naturalista. Alla prima domanda la risposta sarebbe affermativa, mentre la seconda richiede uno studio più approfondito. ${ }^{5}$ Uno dei protagonisti alla fine de $I l$ paese di cuccagna, con le ultime "cento lire [...] chiuse nel suo vuoto portafoglio [...] sentiva come un calore crescente, poiché quella moneta era veramente l'ultima parola del destino. Non avrebbe trovato più niente: tutto era detto" (I890: 2II). La scrittrice in poche parole ci comunica il fortissimo potere del denaro di trasformare la vita di un individuo e questa metafora del denaro come "parola del destino" sembra avere un carattere piuttosto innovativo. Una metafora monetaria ancora più originale e sorprendente viene presentata da Federigo Tozzi, uno scrittore che abbandona il paradigma naturalista, in Con gli occhi chiusi:

gli mostrò tutti i soldi riscossi: - Li vedi? Son come noi uomini: chi è fatto in un modo e chi in un altro. Questo è stato battuto con il martello, e appena si conosce com'è. Quest'altro è piegato, come se uno è zoppo; quest'altro lo volevano bucare, come se tu dài una coltellata a qualcuno o la dànno a te; e questo è consumato tanto che pesa metà; è un povero come me; e me lo beverò per il primo, perché non mi ci faccia pensare. A rivederci. (I9I9: 58).

Il personaggio tozziano propone in questo caso la similitudine "i soldi sono come uomini" per affermare il valore uguale di tutti gli esseri umani. Come le monete del campagnolo toscano, siamo tutti diversi dal punto di vista fisico, ma abbiamo lo stesso valore come esseri umani. In questo modo Tozzi capovolge la metafora ponendo il valore umano sullo stesso piano del valore monetario, dimostrando il potenziale conoscitivo e liberatorio della metafora poetica.

\section{Note}

I. Si intende inoltre studiare la funzione di questi motivi nella struttura narrativa dei testi, la loro presenza nei vari generi romanzeschi e le visioni del mondo dei narratori che risultano dal loro trattamento dei problemi economici.

2. I moderni strumenti informatici permettono di quantificare la presenza delle parole chiave nei corpus. Solo una parte minore delle parole quali "denaro", "soldi", "quattrini" e "lira" fanno parte delle espressioni metaforiche. In L'eredità Ferramonti "denaro" e "quattrini" appaiono cinquanta volte, mentre 
per Verga e Serao i numeri corrispondenti sono i i9 e 230. La spiccata differenza può in parte essere spiegata dal fatto che il romanzo di Chelli è lungo circa la metà delle altre due opere veriste (o per citare i numeri esatti, i tre romanzi contengono 59532, I09246 rispettivamente I 44402 parole).

3. Lakoff e Johnson parlano di "metaphorical concepts" e "metaphorical linguistic expressions" nel loro studio fondamentale Metaphors We Live By (I980: 7), ma in seguito si useranno i termini "metafore concettuali" e "metafore linguistiche" per indicare gli stessi concetti. Inoltre, si segue la convenzione introdotta dai due studiosi americani di indicare le metafore concettuali con lettere maiuscole.

4. Il carattere universale, $\mathrm{o}$, invece, culturalmente specifico delle metafore è attualmente un ampio campo di ricerca. Rimanendo nell'ambito delle metafore monetarie, si può segnalare, oltre al contributo di O'Connor (I998), quello di Silaški e Kilyeni, "The MONEY IS A LIQUID Metaphor in Economic Terminology - a Contrastive Analysis in English, Serbian and Romanian" (20I I).

5. Secondo uno studio influente di David Lodge (r977), il romanzo modernista e la poesia fanno maggior uso delle metafore rispetto al romanzo realista dell'epoca vittoriana, dove prevale il procedimento metonimico nel senso di Jakobson. Semino e Steen vedono alcune difficoltà nell'approccio di Lodge, pur accettando la validità delle sue osservazioni (2008: 238).

\section{Riferimenti}

Casadei, Federica. 1996. Metafore ed espressioni idiomatiche. Uno studio semantico sull'italiano. Roma: Bulzoni.

Chelli, Gaetano Carlo. 20ıо [ı884]. L'eredità Ferramonti. Roma: Avagliano Editore.

Foucault, Michel. 2006 [1966]. Le parole e le cose. Un'archeologia delle scienze umane. Milano: BUR Saggi.

Johnson, Jerah. I966. “The Money=Blood Metaphor, I300-I800”. The Journal of Finance, 2I:I. II9-I 22

Lakoff, George \& Mark Johnson. 1980. Metaphors We Live By. Chicago: University of Chicago Press.

Lakoff, George \& Mark Turner. 1989. More than Cool Reason. A Field Guide to Poetic Metaphor. Chicago: University of Chicago Press.

Lodge, David. 1977. The Modes of Modern Writing: Metaphor, Metonymy, and the Typology of Modern Literature. Ithaca, N.Y.: Cornell University Press. 
O’Connor, Kathleen Therese. I998. "Money and Finance as Solid, Liquid, and Gas in Spanish”. Metaphor and Symbol, I 3:2. I4 I-I 57.

Poggi, Gianfranco. I998. Denaro e modernità. La "Filosofia del denaro» di Georg Simmel. Bologna: Mulino.

Semino, Elena \& Gerard Steen. 2008. “Metaphor in Literature”. In: Gibbs, Raymond (ed.). The Cambridge Handbook of Metaphor and Thought. New York: Cambridge University Press.

Serao, Matilde. I 890. Il paese di cuccagna. <http://www.liberliber.it/mediateca/libri/s/serao/il_paese_di_cuccagna/pdf/il_pae_p.pdf>

Silaški, Nadežda \& Annamaria Kilyeni. 20ı ı. “The MONEY IS A LIQUID Metaphor in Economic Terminology - a Contrastive Analysis in English, Serbian and Romanian”. Professional Communication and Translation Studies, 4:I-2. 63-72.

Tozzi, Federigo. I994 [r919]. Con gli occhi chiusi. Ricordi di un impiegato. Milano: Feltrinelli.

Verga, Giovanni. I994 [I889]. Mastro-don Gesualdo. Roma: Newton Compton editori. 